2018-09

\title{
Impact of Video File Format on Quality of Experience (QoE) of Multimedia Content
}

\section{Laghari, A}

http://hdl.handle.net/10026.1/11999

\subsection{7/s13319-018-0191-x \\ 3D Research \\ Springer Verlag}

All content in PEARL is protected by copyright law. Author manuscripts are made available in accordance with publisher policies. Please cite only the published version using the details provided on the item record or document. In the absence of an open licence (e.g. Creative Commons), permissions for further reuse of content should be sought from the publisher or author. 


\title{
Impact of Video File Format on Quality of Experience (QoE) of Multimedia Content
}

\author{
Asif Ali Laghari*, Hui He*, Asiya Khan, Sajida Karim \\ School of computer Science and Technology, Harbin Institute of Technology, Harbin, China \\ University of Plymouth \\ Emai: asiflaghari,hehui[@hit.edu.cn], asiya.khan@plymouth.ac.uk, sajada.abbasi@ salu.edu.pk
}

\begin{abstract}
:
Video file formats are used to record, play and store videos according to heterogeneous platforms; devices and online streaming on networks vary by speed. Video formats are different codecs and resolution from each other and also vary in display quality and storage size which also differentiate the quality of experience (QoE) of users. This paper presents the subjective QoE of end user's for different video file format varied according to resolution quality and bitrate. Videos of most popular formats which are used for online streaming was downloaded from YouTube and stored on the local system for play for subjects to perceive the video quality. Subjective QoE experiments were conducted by questionnaires given to subjects to perceive video quality and assign ratings. Results show high ratings for FLV 240P and WebM 360P for low resolution videos where high ratings for the MP4 video for 720P and 2160P high resolutions.
\end{abstract}

Keywords: Video file formats, Quality of Experience (QoE), Video Quality, Resolution

\section{Introduction:}

Multimedia streaming is a popular service on the Internet and overall $31 \%$ of traffic is used for Internet video streaming [1]. Internet speed is diverse according to bandwidth limitation, country local network, Internet service provider's speed (ISP) and package plan of the user, so it is difficult to access high definition (HD) videos via a slow network with different devices. Different video codecs (formats) were developed to compress the video and reduce size for streaming on the Internet with high quality video [2]. In the early 2000s flash videos were used to play videos in the browsers and adobe flash player was a tool to support play video via real time message protocol (RTMP) [3]. FLV files were encoded with codecs like the Sorenson Spark or VP6 video compression formats and contains video bit streams which are a proprietary variant of the H.263 video standard [4].

The early era of mobile devices did not support FLV properly, so in 2000 3GP video file format was developed by International Telecommunication Union (ITU) in a meeting of telecommunication association to globally implement video file format for mobile streaming [5]. 3GP has low quality video due to low bitrate, screen resolution and frame rate with high compression for support on the small display of mobile devices and low network speed of $2 \mathrm{G}$. the $3 \mathrm{GP}$ file format store video files as MPEG-4 version 2, H.263 or MPEG-4 version 10 advanced video codec (AVC/H.264) and audio streams as AMR-NB, AMR-WB, AMR-WB+, AAC-LC, HE-ACC v or enhanced accplus (HE-ACC v2) $[6,7]$.

MP4 MPEG-4 (motion pictures expert group) is based on the quick time file format and it was introduced in 2001 for public use [8]. MP4 file format was developed by International organization of standards (ISO) with extensions such $. m p 4, . m 4 a, m 4 p, m 4 b, . m 4 r$ and .m4v with standard number ISO/IEC 14496-14. Different versions of MP4 file format were introduced with different compression codec such H.263, H.264 and H.265 for providing better video quality with compressed file size [9]. The purpose of launching different versions with a different codec to compress the file 
to transfer on the networks for multimedia streaming to remote users but the limitation of bandwidth is the main consideration during the development $[10,11]$.

WebM initially launched on May 18, 2010, sponsored by Google and license under the distribution of BSD [12, 13 14]. WebM is audiovisual file format and alternative use of HTML5. This supports Google Chrome, Opera and Mozilla Firefox and Internet Explorer required third party software to WebM files [15].

Online streaming of HD video is still concerns for service providers (SPs) due file format of videos used by SP and network speed of user's connection. SPs used user feedback system to get user reviews about video quality and provider better service to them. The quality of experience (QoE) term is used for assessment of user's opinion about the video quality and service they receive [16]. "QoE provides an assessment of human expectations, feelings, perceptions, cognition and satisfaction with respect to a particular product, service or application" [17]. The definition of QoE is given in [18] "the degree of delight or annoyance of the user of an application or service". Further, QoE defined in general perspective "QoE is a measurement of customer satisfaction or customer performance depends on the objective or subjective measure of using any service or product" [19]. HD videos take additional time to load into a webpage as compare to the low quality, the big size of the video also utilize more storage space on the cloud/server and requires more network bandwidth to transfer from cloud/server to client. The main contribution of this paper is to assess the QoE of the end user on the different video file format and quality to analyze which file format and resolution are suitable for video streaming over cloud/server video hosting website which fulfills the user needs.

During the QoE assessment experiments, we use subjective QoE method to assess user satisfaction level for different video file formats and resolutions. Solution provides the acceptable quality level (resolution, bitrate, frame rate) of video file formats for users, which provided by different developers for online streaming. During the research work, we repeat experiments with four video file formats having different quality $4 \mathrm{~K}(2160 \mathrm{P}), 720 \mathrm{P}$ HD, 360P, and 240P which were taken from YouTube and stored on the local system. Downloaded videos were played for the user to perceive videos to assign ratings and examine the characteristics and effect on the $\mathrm{QoE}$ of video quality.

Our paper is organized into 5 sections, in section 2 we provide the literature review and section 3 is based on laboratory experiments. Section 4 provides results and discussion; finally, in section 5 we conclude our work.

\section{Literature Review:}

Comparative research works on different video formats were given by researcher in past and one such work given by Mustafa et al. [4]. During the research, the algorithm was proposed to compare the performance of MPEG4, FLV and 3GP by considering wireless network parameters. Metadata of files were calculated before and after transmitted on the network, algorithm use peak signal to noise ratio (PSNR) and signal to noise ratio (SNR) to calculate variation and fluctuation of files. Results show that MPEG4 have highest variance and fluctuation and 3GP have lowest where FLV have middle level. The research proves that $3 \mathrm{GP}$ provide better results compare to MPEG and FLV file formats.

Research work provided by Gohil and Pandya [20] on the compression of video file formats. The comparative work based on the conversion of one original file format into other video file formats such as MP4, AVI, 3GP, WMV, MKV, MPG, VOB, MOV and FLV. The comparative work based on the compression (conversion) time and file size with high video quality. The results were given in comparative study that different format has different time of conversion from the original file and different file size.

Rogge et al. presented a reference model for the future multimedia format for timing issue [21]. This reference model document is based on the ten rules such as 29 interval relationships, fine-grained model, timestamp relationships versus original timing information, extensibility, interaction, reusability, presentation neutral representation, adaptability, real-time application support and QoS parameters. This model applied on multimedia formats for performance comparisons such as Advanced Streaming, QuickTime, RealVideo, Shockwave and MPEG-4. Results show that SMIL is the only document which supports all 29 temporal relationships put forward in the reference model. Results also a proof difference between non-streaming and streaming video formats and streaming video formats do not support partial reuse. Only MPEG-4 has an exception from this rule because mostly MPEG-4 is used for wide 
range of applications. Compressed formats are evolving towards the delivery formats and RealMedia now supports limited delivery functionality through the use of SMIL. Newly developed multimedia video formats support a media schema that can be sent in advance and function as lookup library for the actual raw media data. Only MPEG-4 provides better QoS support compared to the other multimedia video formats, which describes some parameters and their needs concerning bandwidth.

In the past, only QoE of different video resolution was assessed considering network parameters form long distance and nearby locations servers [22] and QoE of MP4 file was assessed regarding social cloud compression parameters [23], but QoE was never considered for the comparative study of file formats with different resolution.

\section{QoE Assessment Experiment:}

QoE assessment of multimedia formats based on the four videos having different file formats (extensions) and different resolutions. Videos were downloaded from YouTube in FLV, WebM, MP4 and 3GP formats and resolutions were 240P, 360P, 720P and 4K (2160) [24, 25, 26, 27] by using download website of third party [28, 29]. Resolution and frame rate of videos are according to standard given by YouTube streaming, which is varying according to devices such as smartphones (small screen) and desktop (big screen) [30].

During the QoE measurement of experimental data, 83 users participated from the department of computer science and information technology and few were from other departments of nontechnical fields such as social sciences, mostly users belonged to undergraduate studies and rest of them were postgraduates. Subject's age was between 21 to 33, there were 47 males and 36 were females and belong to Chinese and Russian nationalities. A questionnaire was given to users and guided them to provide profile information and perceive video quality of different video file format as same resolution and assign a rating for video quality which was played on the screen. We follow the recommendations of ITU-R and ITU-T Rec. for display of video [31, 32]. The display setting for the perception of video for users 21-inch LCD display was used, which has a resolution of $1280 \times 800$ and for $4 \mathrm{k}$ video we use 55 inches have $3840 \times 2160 \mathrm{HD}$ resolution, where videos were played in sequence. We used absolute category rating (ACR) scale to evaluate the quality of videos, which is given in Table 1 and experimental methodology is shown in Figure 1 [33]. The average value of opinion ratings over all the subjects is called the mean opinion score (MOS) [34, 35]. The cumulative distribution of opinion score are given in Tables and Figures in results and discussion section.

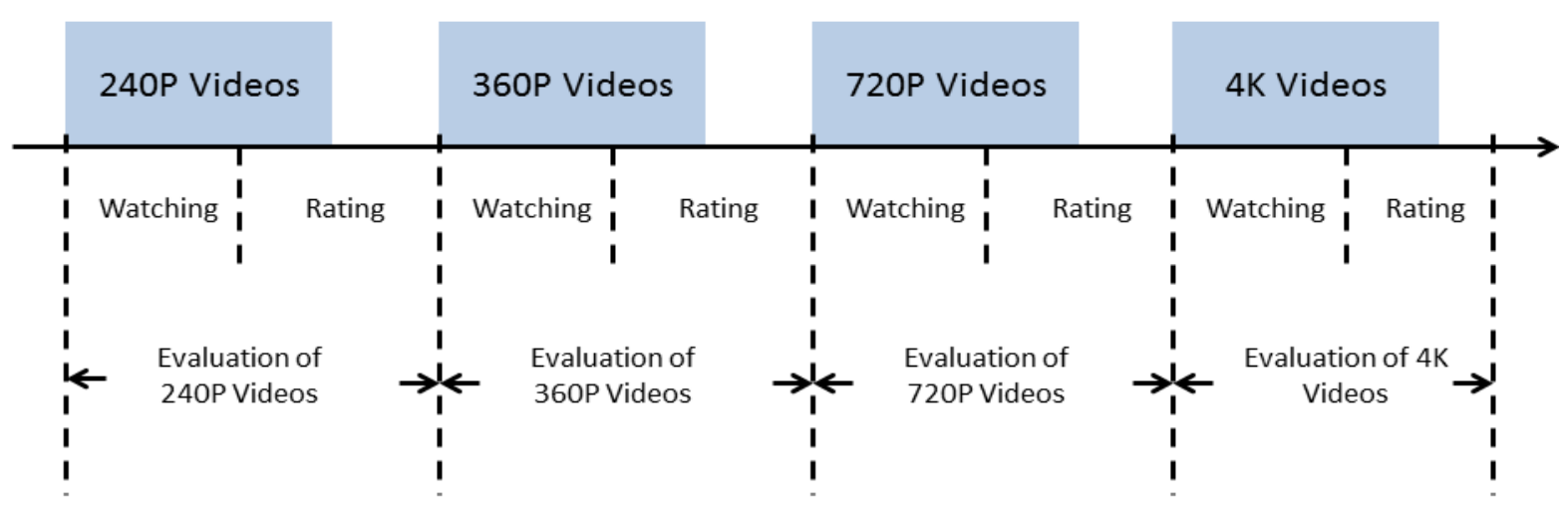

Figure 1. ACR Experimental methodology

Table 1. ACR Scores 


\begin{tabular}{|c|c|}
\hline Score & Quality \\
\hline 5 & Excellent \\
\hline 4 & Good \\
\hline 3 & Fair \\
\hline 2 & Poor \\
\hline 1 & Bad \\
\hline
\end{tabular}

\section{Results and Discussion:}

File viewer lite and VLC (version 3.0.2 Vetinari) players were used for open and play videos for user perception [36, 37, 38]. During QoE test videos were used vary by file formats and resolution standard given by developers, the purpose of using different parameters to collect user mean opinion to analyze which file format provides better QoE at resolution compared to others. Report log of all videos was generated by using MediaInfo software tool (version, 18.03.1) developed by MediaArea.net SARL [39]. The MediaInfo tool decodes all information of videos such as file format, resolution, total bitrate, frame rate, audio bitrate, storage size and playing time.

240P videos were played for users in FLV, WebM, MP4 and 3GP formats to watch and assign mean opinion score (MOS) according to the quality they perceive. ITU provides standards known as Mean Opinion Score (MOS) to collect subjective responses from users [31]. The technical parameters of 240P video for each format are given in Table 2. Figures 2 to 5 provides snapshots of playing video with a slight quality difference. Results show in Table 3 and Figure 6 that user assign WebM high ratings as compared to other video file formats because of the high total bitrate of video and audio which also increase the storage size of the file. 3GP format has lowest ratings because it does not provide good quality of video due to small frame width and low total bitrate as compared to other formats. The 3GP is mobile based format it provides better quality video on the small screen and smooth online streaming to due low bitrate.

Table 2. 240P video parameters

\begin{tabular}{|c|c|c|c|c|}
\hline $\begin{array}{c}\text { Technical } \\
\text { parameters }\end{array}$ & FLV & WebM & MP4 & 3GP \\
\hline Frame width & 426 & 426 & 426 & 320 \\
\hline Frame height & 240 & 240 & 240 & 240 \\
\hline Video bitrate & - & - & $2409 \mathrm{kbps}$ & $234 \mathrm{kbps}$ \\
\hline Total bitrate & $295 \mathrm{kbps}$ & $784 \mathrm{kbps}$ & 29.97 frames/sec & 29.97 frames/sec \\
\hline Frame rate & 29.97 frames/sec & 29.97 frames/sec & $126 \mathrm{kbps}$ & $32 \mathrm{kbps}$ \\
\hline Audio bitrate & $18.8 \mathrm{kbps}$ & $-\mathrm{kbps}$ & $1.97 \mathrm{MB}$ & $1.89 \mathrm{MB}$ \\
\hline Storage size & $1.99 \mathrm{MB}$ & $6.3 \mathrm{MB}$ & $67 \mathrm{sec}$ & $67 \mathrm{sec}$ \\
\hline Playing length & $67 \mathrm{sec}$ & $67 \mathrm{sec}$ & Mp4 (Base Media) & $3 \mathrm{GP}$ Media \\
& & & & \\
\hline Codec Type & Advanced Video & WebM (Version 2)
\end{tabular}




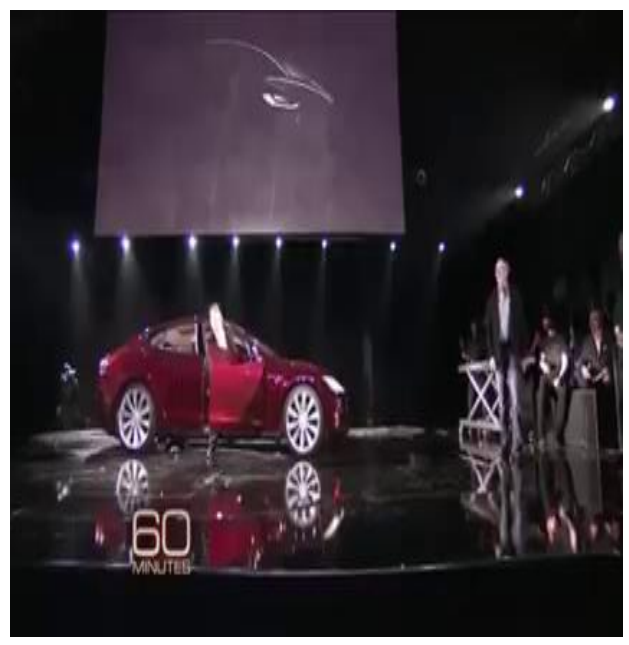

Fig 2. 240 FLV snapshot

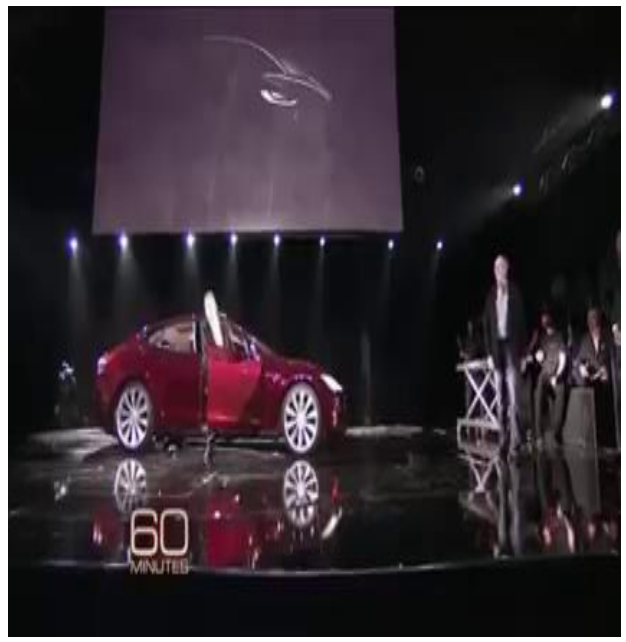

Fig 4. 240 MP4 snapshot

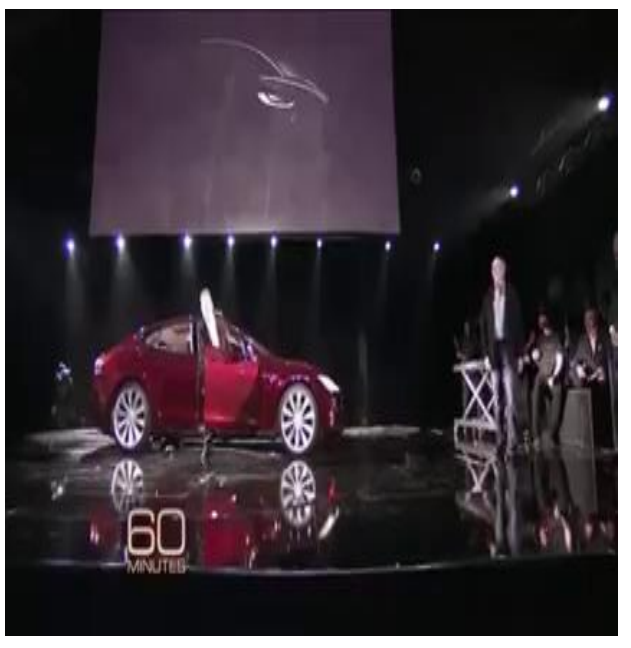

Fig 3. 240 WebM snapshot

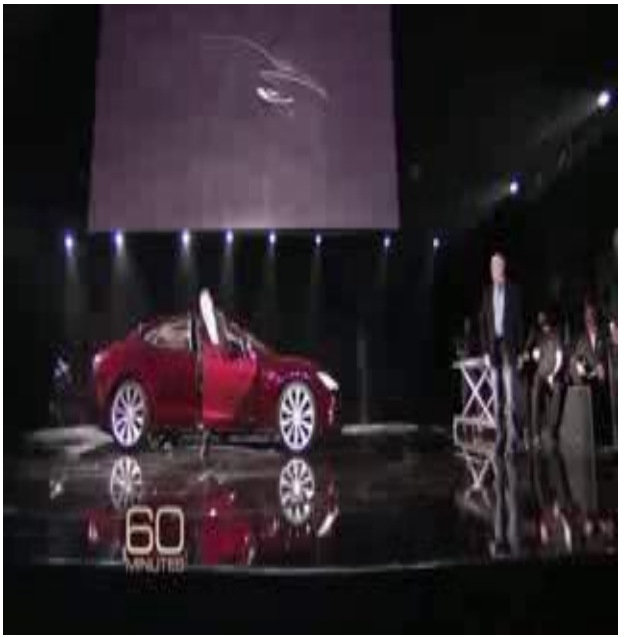

Fig 5. 240 3GP snapshot

Table 3. User MOS of 240P

\begin{tabular}{|c|c|}
\hline Video format & Mean Opinion Score \\
\hline FLV & 2.7 \\
\hline WebM & 3.4 \\
\hline MP4 & 2.5 \\
\hline 3GP & 1.8 \\
\hline
\end{tabular}


Multimedia Format Comparison 240P

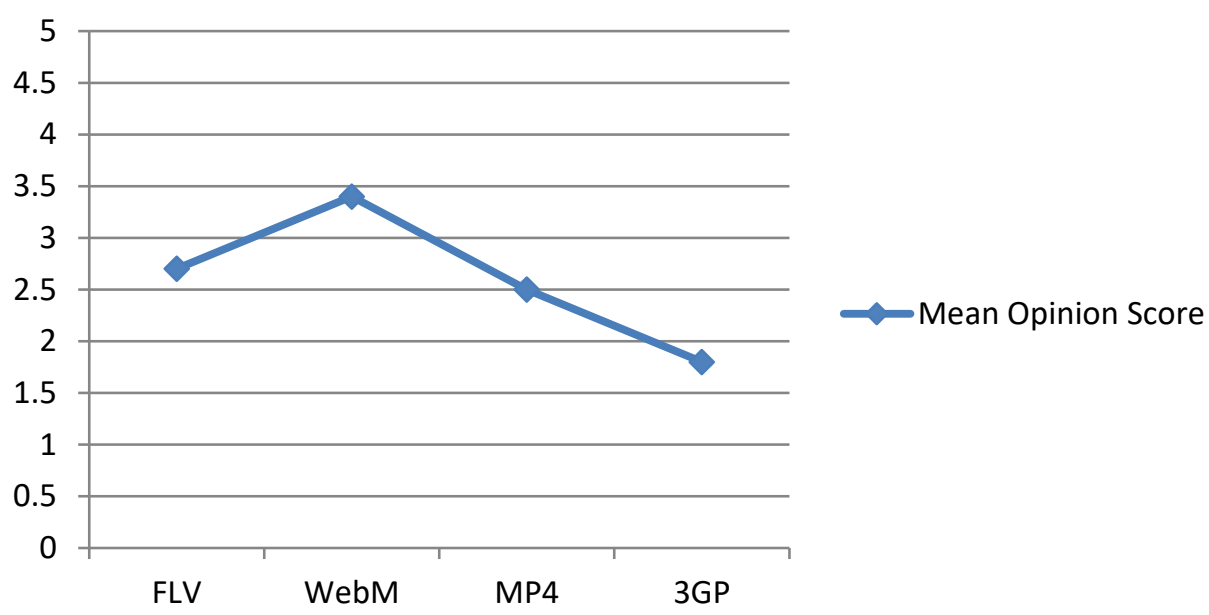

Figure 6. Comparison of 240P video formats

The 360P videos were selected in different formats for quality comparative analysis. 360P required faster network as compared to $240 \mathrm{P}$ due to high video quality and file size. 360P have overall bitrate, video bitrate and screen resolution are high compared to $240 \mathrm{P}$ videos which provide clearer and high quality videos so user assigned high MOS. The technical information is shown in Table 4 that WebM format have big file size as compare to other formats because it has high video bitrate $590 \mathrm{kbps}$, total bitrate $756 \mathrm{kbps}$ with $128 \mathrm{kbps}$ which more increased from other video file formats. Results show in Table 5 and Figure 11 that user assigned high ratings for WebM and MP4 video where 3GP has lowest ratings because $3 \mathrm{GP}$ has more distorted video compare to other file formats which are shown in Figure 7 to 10 respectively. The $3 \mathrm{GP}$ format in mobile devices provides good video quality on small screen and required low network bandwidth for transfer from server to client where WebM required high speed network for transfer because of big file size.

Table 4. 360P video parameters

\begin{tabular}{|c|c|c|c|c|}
\hline $\begin{array}{c}\text { Technical } \\
\text { parameters }\end{array}$ & FLV & WebM & MP4 & 3GP \\
\hline Frame width & 640 & 640 & 640 & 640 \\
\hline Frame height & 360 & 360 & 360 & 360 \\
\hline Video bitrate & $479 \mathrm{kbps}$ & $590 \mathrm{kbps}$ & $490 \mathrm{kbps}$ & $350 \mathrm{kbps}$ \\
\hline Total bitrate & $595 \mathrm{kbps}$ & $756 \mathrm{kbps}$ & $588 \mathrm{kbps}$ & $365 \mathrm{kbps}$ \\
\hline Frame rate & 25 frames/sec & 25 frames/sec & 25 frames/sec & 18 frames/sec \\
\hline Audio bitrate & $93.8 \mathrm{kbps}$ & $128 \mathrm{kbps}$ & $96 \mathrm{kbps}$ & $12.8 \mathrm{kbps}$ \\
\hline Storage size & $2.63 \mathrm{MB}$ & $3.34 \mathrm{MB}$ & $2.60 \mathrm{MB}$ & $1.62 \mathrm{MB}$ \\
\hline Playing length & $37 \mathrm{sec}$ & $37 \mathrm{sec}$ & $37 \mathrm{sec}$ & $37 \mathrm{sec}$ \\
\hline Format (media type) & Advanced Video & WebM (Version 2) & Mp4 (Base Media / & $3 \mathrm{GP}$ Media \\
\hline
\end{tabular}




\begin{tabular}{|l|l|l|l|c|}
\hline & Codec (AVC) & & version 2) & Release 4 \\
\hline
\end{tabular}

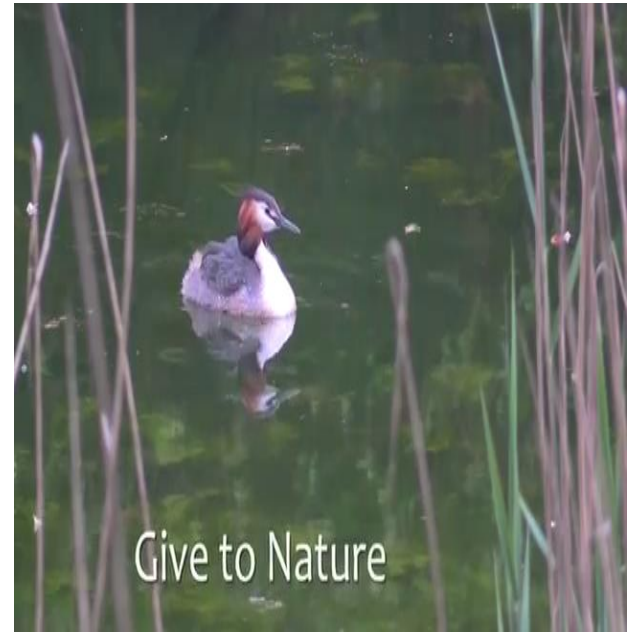

Fig 7. 360 FLV snapshot

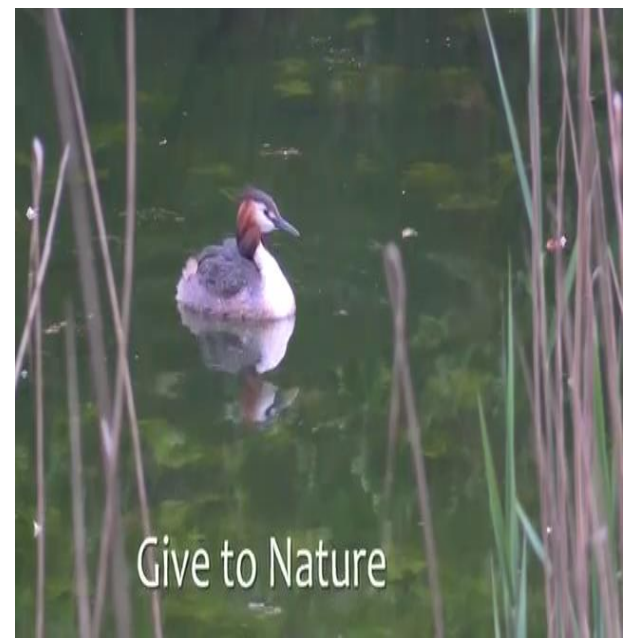

Fig 9. 360 MP4 snapshot

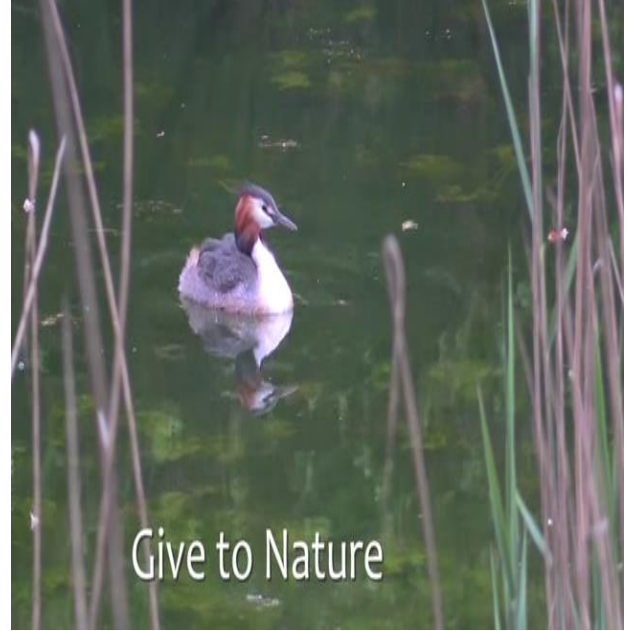

Fig 8. 360 WebM snapshot

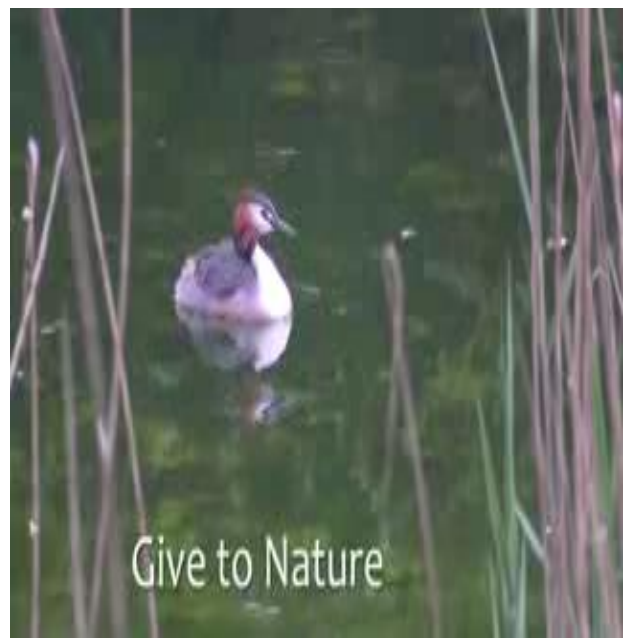

Fig 10. 360 3GP snapshot

Table 5. User MOS of 360P

\begin{tabular}{|c|c|}
\hline Video format & Mean Opinion Score \\
\hline FLV & 3.2 \\
\hline WebM & 4.2 \\
\hline MP4 & 3.8 \\
\hline 3GP & 2.3 \\
\hline
\end{tabular}




\section{Multimedia Format Comparison 360P}

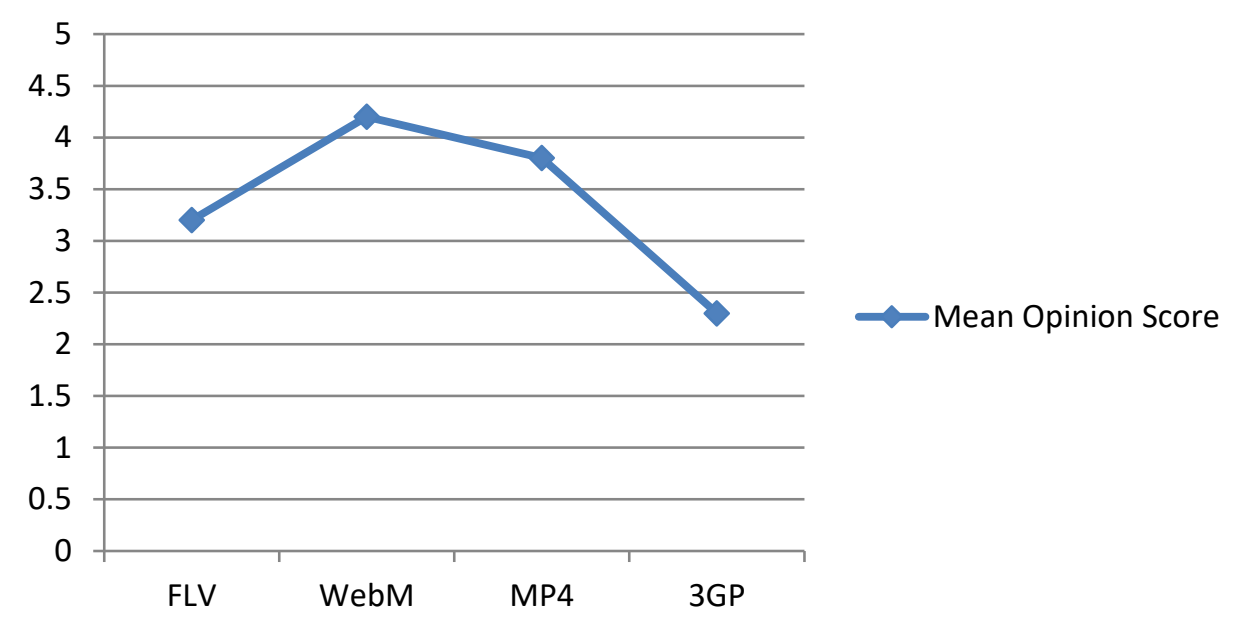

Figure 11. Comparison of 360P video formats

FLV and 3GP do not support resolution of 720P HD or higher quality videos so only we consider WebM and MP4 720P and 4K videos for QoE assessment experiment [40]. 720P HD video selected in WebM and MP4 file formats for QoE assessment. The technical information is given in Table 6 show that WebM is more compressed file format and MP4 is less compressed because of high file size, total bitrate which required more network bandwidth for online video streaming. The result shown in Table 7 and Figure 14 that user assigned high ratings for MP4 video as compare to WebM because user assesses that MP4 video provides better quality as compare to WebM. Figure 12 and 13 shows the quality comparison snapshots of WebM and MP4 during the playing of videos.

Table 6. 720P video parameters

\begin{tabular}{|c|c|c|}
\hline $\begin{array}{c}\text { Technical } \\
\text { parameters }\end{array}$ & WebM & MP4 \\
\hline Frame width & 1280 & 1280 \\
\hline Frame height & 720 & 720 \\
\hline Total bitrate & $1335 \mathrm{kbps}$ & $1748 \mathrm{kbps}$ \\
\hline Frame rate & 29.97 frames/sec & 29.97 frames/sec \\
\hline Storage size & $10.3 \mathrm{MB}$ & $13.5 \mathrm{MB}$ \\
\hline Playing length & $64 \mathrm{sec}$ & 64 sec \\
\hline Format (media type) & WebM (Version 4/2) & $\begin{array}{c}\text { Mp4 (Base Media / } \\
\text { version 2) }\end{array}$ \\
\hline
\end{tabular}




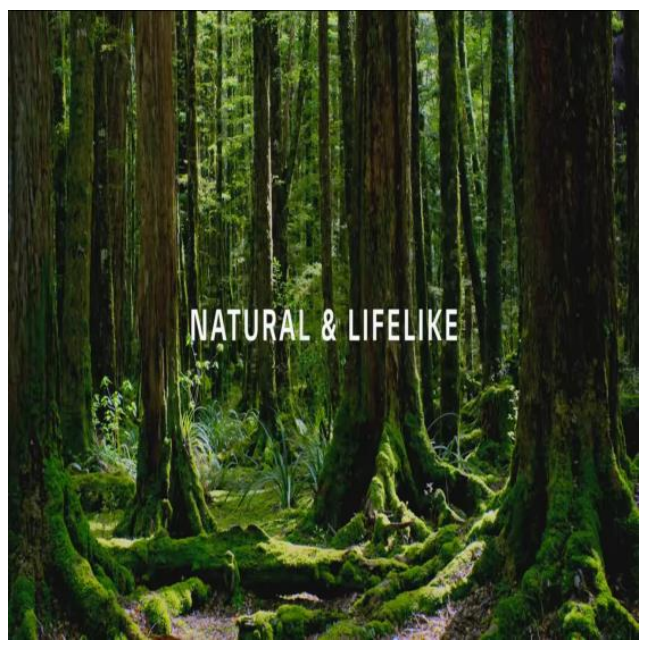

Fig 12. 720 WebM snapshot

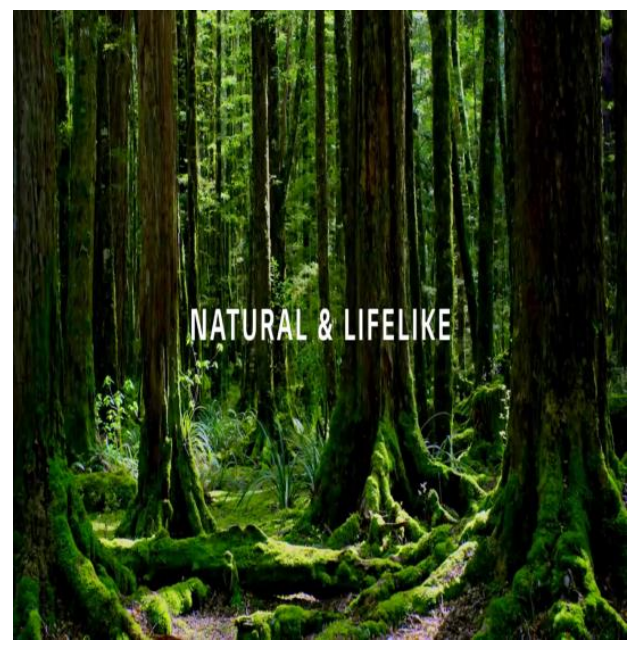

Fig 13. 720 MP4 snapshot

Table 7. User MOS of 720P

\begin{tabular}{|c|c|}
\hline Video format & Mean Opinion Score \\
\hline WebM & 4.4 \\
\hline MP4 & 4.5 \\
\hline
\end{tabular}

Multimedia Format Comparison 720P

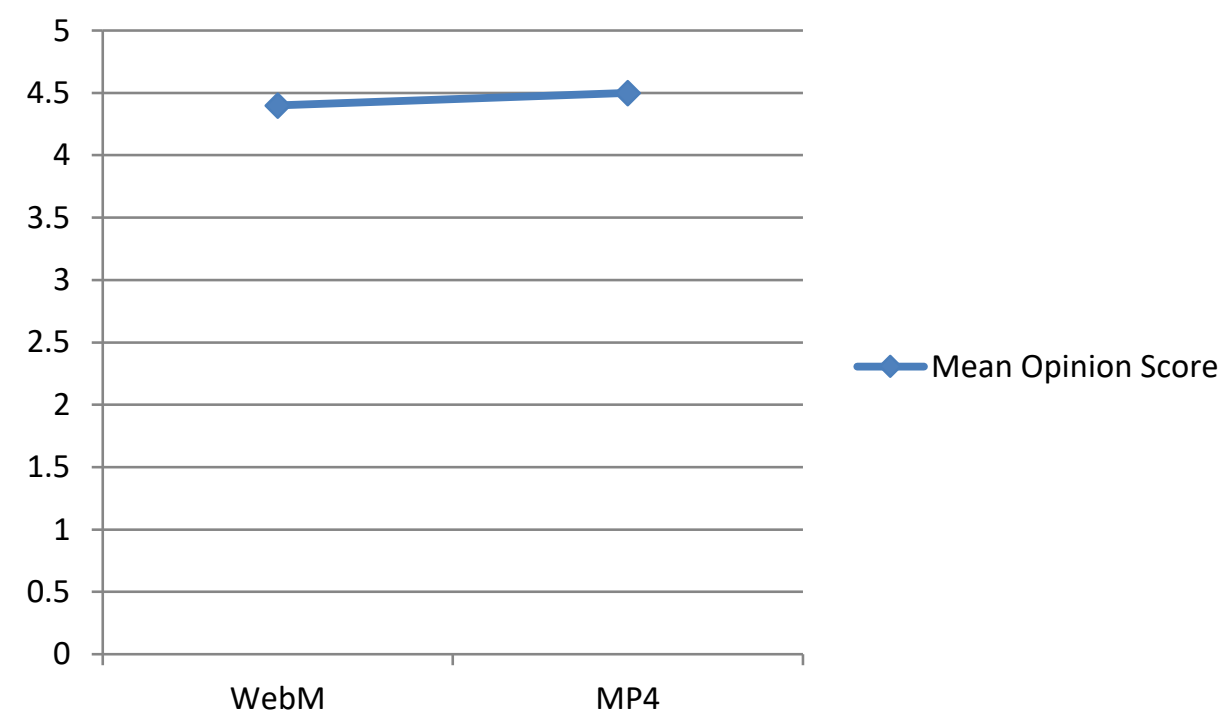

Figure 14. Comparison of $720 \mathrm{P}$ video formats

$4 \mathrm{~K}(2160 \mathrm{P})$ videos were played for the user to watch and perceive the video quality and assign MOS score for the particular file format. Technical information of video is given in Table 8 which shows those MP4 formats have double total bitrate and also file size. WebM more compressed file format compare to MP4 in sense of storage and total bitrate 
that required low network bandwidth for video streaming. Results given in Table 9 and Figure 17 show that user assigned high rating to MP4 video and slightly less to WebM this proof that WebM file format almost provides same video quality with high compression of the file which saves the storage size and as well as network resources for transmission. Figure 15 and 16 shows the quality comparison snapshots of WebM and MP4 during the playing of videos.

Table 8. 4K (2160p) video parameters

\begin{tabular}{|c|c|c|}
\hline $\begin{array}{c}\text { Technical } \\
\text { parameters }\end{array}$ & WebM & MP4 \\
\hline Frame width & 3840 & 3840 \\
\hline Frame height & 2160 & 2160 \\
\hline Total bitrate & $12.6 \mathrm{MB} / \mathrm{s}$ & $30.6 \mathrm{MB} / \mathrm{s}$ \\
\hline Frame rate & $29.97 \mathrm{frames} / \mathrm{sec}$ & $29.97 \mathrm{frames} / \mathrm{sec}$ \\
\hline Storage size & $106 \mathrm{MB}$ & $258 \mathrm{MB}$ \\
\hline Playing length & $70 \mathrm{sec}$ & $70 \mathrm{sec}$ \\
\hline Format (media type) & WebM (Version 4/2) & Mp4 (Base Media) \\
\hline
\end{tabular}

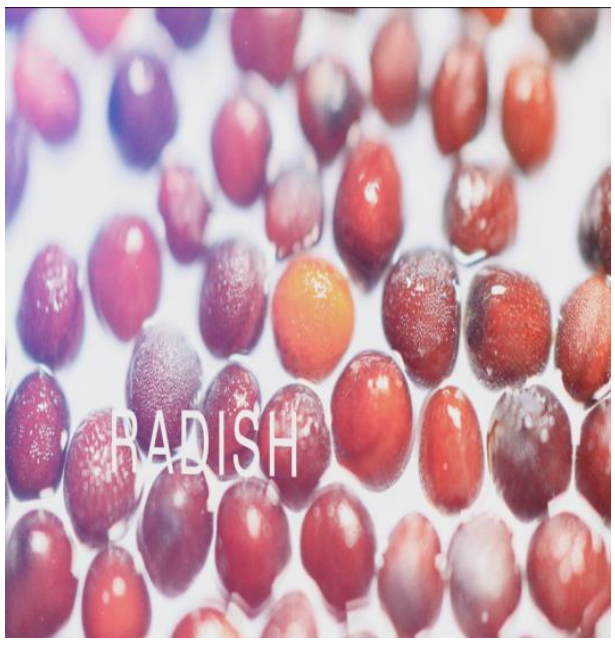

Fig 15. 4K WebM snapshot

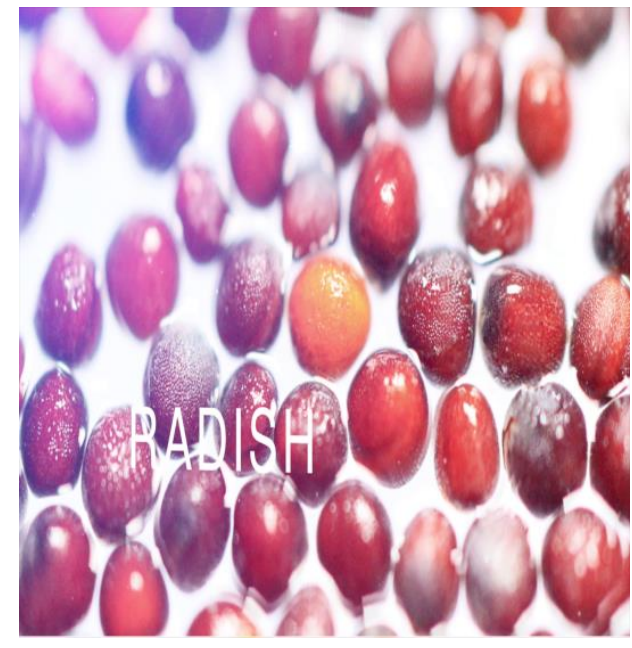

Fig 16. 4K MP4 snapshot

Table 9. User MOS of $4 \mathrm{~K}$

\begin{tabular}{|c|c|}
\hline Video format & Mean Opinion Score \\
\hline WebM & 4.8 \\
\hline MP4 & 4.9 \\
\hline
\end{tabular}




\section{Multimedia Format Comparison 4K}

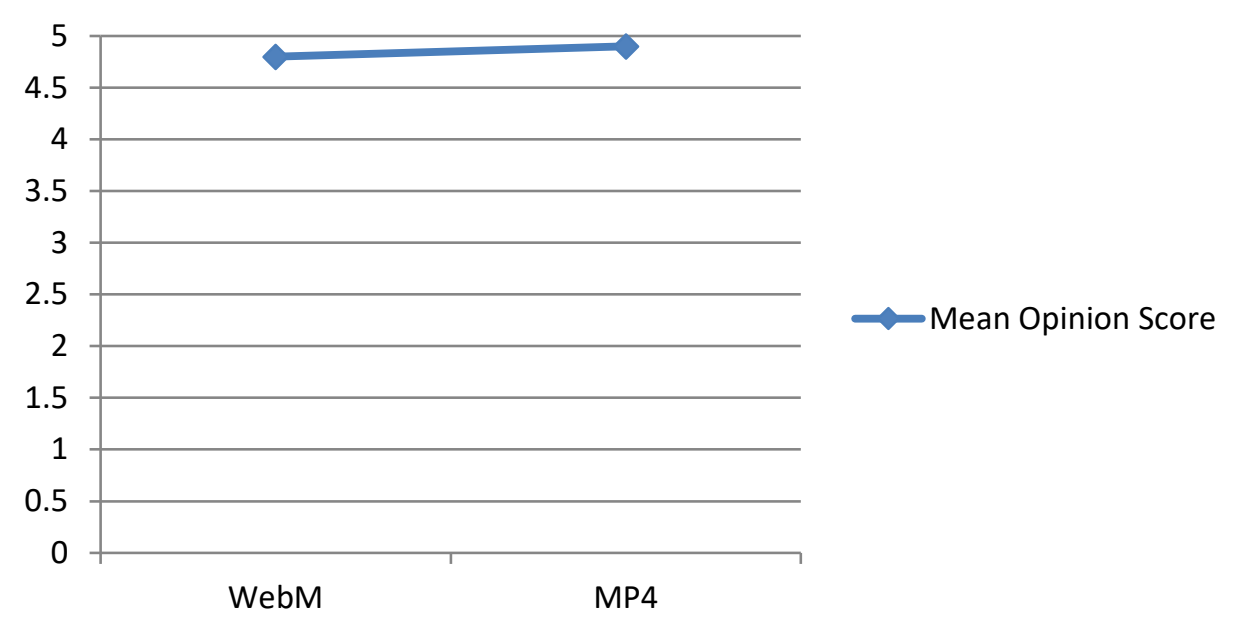

Figure 17. Comparison of $4 \mathrm{~K}$ video formats

Comparison of all multimedia formats is given in Figure 18, where bar graph shows the user assigned ratings to multimedia formats. Currently default codec load by YouTube for online streaming via Google Chrome browser is WebM, which can checked by clicking on "stat for nerds" option on playing video, but in this research users provide high quality ratings MP4 videos for 720P and 4K videos and for low quality video FLV 240P and WebM 360P have high ratings.

\section{Multimedia Format Comparison}

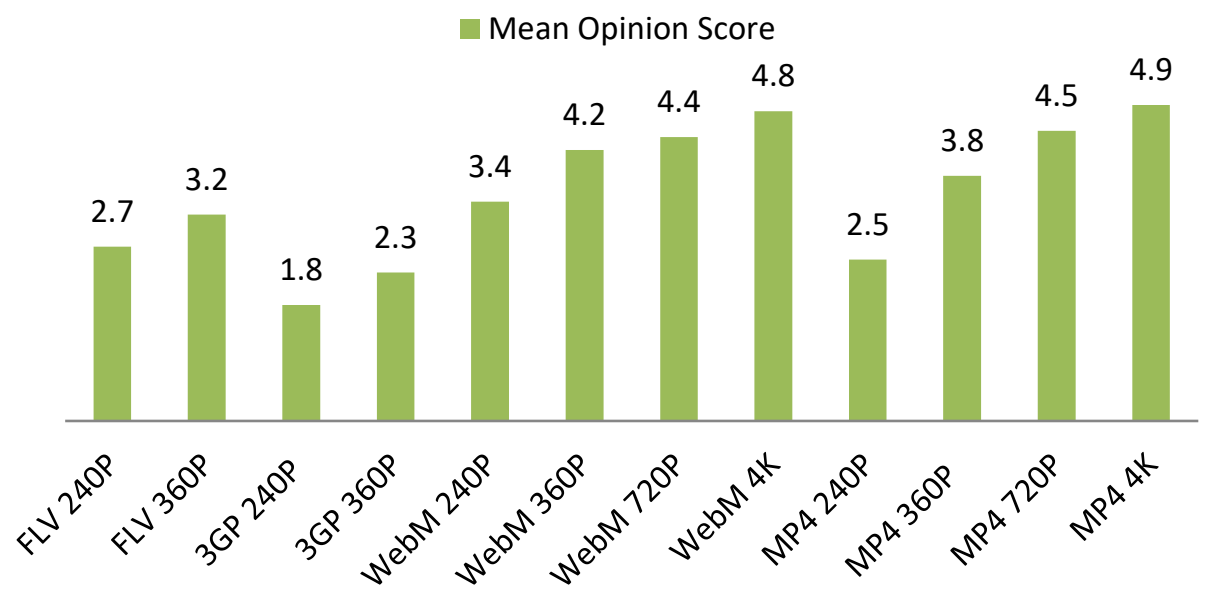

Figure 18. Overall comparison of video formats

\section{Conclusion:}

In this paper, we conduct several QoE assessment experiments on 4 different multimedia format such as FLV, 3GP, WebM and MP4 with quality and resolutions of 240P, 360P, 720P and 2160P respectively to measure user satisfaction 
level on quality for video format. The results comprised in this paper show that user provides high ratings for FLV 240P and WebM 360P for low resolution videos where high MOS for MP4 video for 720P and 2160P high resolutions. Newly developed WebM provide high compression of videos with slightly less user MOS same resolution compared to MP4 which is good for online streaming because high compression reduced file size which required low network speed to transfer from server/cloud to client. This research work provides an assessment of user perception for different video file formats and suitable quality for watching and online streaming, which is beneficial for service providers to use particular format with resolution according to the speed of the network.

Acknowledgment: The work is supported by the National Key R\&D Program of China under grant No. 2017YB0801801, the National Science Foundation of China (NSFC) under grant No. 61472108. Asif Ali Laghari and Professor Hui He are the corresponding authors.

\section{References:}

[1] Networking Index, C.V., (2016). Forecast and methodology, 2016-2021, white paper. San Jose, CA, USA, 1.

[2] Laghari, A.A., He, H., Zardari, S. and Shafiq, M., (2017). Systematic Analysis of Quality of Experience (QoE) Frameworks for Multimedia Services. IJCSNS, 17(5), p.121.

[3] Xiao, Y., Kalyanaraman, R.S. and Yla-Jaaski, A., (2008). Energy consumption of mobile youtube: Quantitative measurement and analysis. In Next Generation Mobile Applications, Services and Technologies, 2008. NGMAST'08. The Second International Conference on (pp. 61-69).

[4] Mustafa, M.A., Elahi, M.M., Hossain, M.A. and Islam, M.M., (2010). Comparative performance analysis of MPEG4, FLV and 3GP multimedia file formats using wireless network parameters. In Computer and Information Technology (ICCIT), 2010 13th International Conference on (pp. 306-311).

[5] Gloe, T., Fischer, A. and Kirchner, M., (2014). Forensic analysis of video file formats. Digital Investigation, 11, pp.S68-S76.

[6] Amon, P., Rathgen, T. and Singer, D., (2007). File format for scalable video coding. IEEE Transactions on Circuits and Systems for Video Technology, 17(9), pp.1174-1185.

[7] Varsa, V. and Curcio, I., (2003). Transparent end-to-end packet switched streaming service (pss); rtp usage model (release 5). 3GPP TR 26.937 V1. 4.0.

[8] McNamee, R., Evans, G. and Frederick, M.R., ROGER B and Ann K Mcnamee Trust U/t/a/d, (2017). Systems and methods for communicating events to users. U.S. Patent 9,788,035.

[9] Tian, L., Wang, H., Zhou, Y. and Peng, C., (2018). Video big data in smart city: Background construction and optimization for surveillance video processing. Future Generation Computer Systems.

[10] Kalampogia, A. and Koutsakis, P., (2018). H. 264 and H. 265 video bandwidth prediction. IEEE Transactions on Multimedia, 20(1), pp.171-182.

[11] Zaidi, S., Bitam, S. and Mellouk, A., (2018). Hybrid error recovery protocol for video streaming in vehicle ad hoc networks. Vehicular Communications, 12, pp.110-126.

[12] Rajan, R., Sekhon, N.K., Kaur, J. and Tiwari, M., (2017). Mobile Computing and Android Support: A.

[13] https://www.webmproject.org/about/ [4/28/2018] 
[14 Kromer, R., (2017). Matroska and FFV1: One File Format for Film and Video Archiving?. Journal of Film Preservation, (96), p.41.

[15] Lopes Goncalves Magalhaes, F., (2017). Intelligent Security System connected to IoT.

[16] Laghari, A.A., He, H., Halepoto, I.A., Memon, M.S. and Parveen, S., (2017). Analysis of Quality of Experience Frameworks for Cloud Computing. IJCSNS, 17(12), p.228.

[17] Laghari, A.A., Laghari, K.U.R., Channa, M.I. and Falk, T.H., (2012). QON: Quality of experience (QoE) framework for network services. In Proceedings of the 4th International Conference on Software Technology and Engineering (ICSTE'12).

[18] Le Callet, P., Möller, S. and Perkis, A., (2013). Qualinet White Paper on Definitions of Quality of Experience (2012). European Network on Quality of Experience in Multimedia Systems and Services (COST Action IC 1003). Version 1.2. Mar-2013.

[19] Laghari, A. A., Sadhayo, I. H., \& Channa, M. I. (2015). Enhanced autonomic networking management architecture (Enama). Engineering, Science \& Technology, 14(1), 9-13.

[20] Gohil, R. and Pandya, V., (2015). A Comparative Study of Different Video Compression.

[21] Rogge, B., Bekaert, J. and Van de Walle, R., (2004). Timing issues in multimedia formats: review of the principles and comparison of existing formats. IEEE Transactions on Multimedia, 6(6), pp.910-924.

[22] Laghari, A.A., He, H., Shafiq, M. and Khan, A., (2016). Assessing effect of Cloud distance on end user's Quality of Experience (QoE). In Computer and Communications (ICCC), 2016 2nd IEEE International Conference on (pp. 500-505).

[23] Laghari, A.A., He, H., Karim, S., Shah, H.A. and Karn, N.K., (2017). Quality of Experience Assessment of Video Quality in Social Clouds. Wireless Communications and Mobile Computing, 2017.

[24] https://www.youtube.com/watch?v=fLeJJPxua3E [240p ] [accessed on 4/13/2018]

[25] https://www.youtube.com/watch?v=76f6J4x87hI [360p] [accessed on 4/13/2018]

[26] https://www.youtube.com/watch?v=27bdrguQxew [720] [accessed on 4/13/2018]

[27] https://www.youtube.com/watch?v=i9RQX1oGtEs [4K] [accessed on 4/13/2018]

[28] https://en.savefrom.net/1-how-to-download-youtube-video/[accessed on 4/13/2018]

[29] https://mediasave.online/ [accessed on 4/13/2018]

[30] https://www.genyoutube.net/formats-resolution-youtube-videos.html [accessed on 4/13/2018]

[311 UIT. U.. 500-11 Methodologv for the subjective assessment of the quality of television pictures. (2002). International Telecommunication Union.

[321 ITU-T RECOMMENDATION, P., (1999). Subjective video quality assessment methods for multimedia applications.

1331 Youn. S.. Baek. S.. Jeong. T. and Lee. C., (2018). Perceptual video quality comparison of various 3D video formats and displays. Displays, 52, pp.21-29. 
Г341 Laghari. A.A.. He. H. and Channa. M.I.. (2018). Measuring Effect of Packet Reordering on Quality of Experience (QoE) in Video Streaming. 3D Research, 9(3), p.30.

[35] Laghari, A.A., He, H. Shafiq, M. \& Khan, A. (2018). Assessment of Quality of Experience (QoE) of Image Compression in Social Cloud Computing. Multiagent and Grid Systems, Vol 14, no. 2, pp. 125-143.

[36] https://windowsfileviewer.com/ [accessed on 5/13/2018]

[37] Solera, M., Toril, M., Palomo, I., Gomez, G. and Poncela, J., (2018). A Testbed for Evaluating Video Streaming Services in LTE. Wireless Personal Communications, 98(3), pp.2753-2773.

[38] https://www.videolan.org/index.html. [Accessed on 5/13/2018]

[39] https://www.videohelp.com/software/MediaInfo [accessed on 5/13/2018]

[40] Hoque, M.A., Siekkinen, M., Nurminen, J.K., Aalto, M. and Tarkoma, S., (2015). Mobile multimedia streaming techniques: QoE and energy saving perspective. Pervasive and Mobile Computing, 16, pp.96-114. 